\title{
Afrontamiento del estrés y bienestar psicológico en estudiantes universitarios de Lima y Huancayo
}

\author{
María Luisa Matalinares \\ Gloria Díaz \\ Ornella Raymundo \\ Deyvi Baca \\ Joel Uceda \\ Juan Yaringaño \\ Universidad Nacional Mayor de San Marcos \\ Lima, Perú
}

Recibido: 1 de abril del 2016 / Aprobado: 20 de julio del 2016

El presente estudio tuvo como objetivo establecer la relación entre los modos de afrontamiento del estrés y el bienestar psicológico. Se evaluó a 934 estudiantes universitarios de Lima y Huancayo con edades entre 16 y 25 años, a quienes se les aplicó la Escala de Modos de Afrontamiento al Estrés (COPE), diseñada por Carver, Scheir y Weintraub (1989), adaptada al contexto peruano, y la Escala de Bienestar Psicológico (SPWB), propuesta por Carol Ryff (1989), adaptada al contexto peruano. Los resultados mostraron que existe una relación directa y significativa entre el modo de afrontamiento del estrés centrado en el problema y la emoción, y el bienestar psicológico, mientras que otros estilos de afrontamiento guardan una relación significativa, pero indirecta.

afrontamiento / estrés / bienestar psicológico / enfoque centrado en el problema

\section{Coping with Stress and Psychological Well-Being in University Students from the Peruvian Cities of Lima and Huancayo}

The purpose of this study is to establish the relationship between stress coping styles and psychological well-being. We examined a sample of 934 university students from the cities of Lima and Huancayo, aged between 16 and 22 years. Participants completed the COPE Inventory and the Ryff's Psychological Well-Being Scales (PWB), both adapted to the Peruvian context. Results show a direct and significant relation between problem-focused and emotion-focused coping strategies and psychological well-being, whereas other coping strategies obtained a significant but indirect relationship.

stress coping strategies / psychological well-being / problem focus / emotion focus

Correo electrónico: maria_luisa93@hotmail.com 


\section{INTRODUCCIÓN}

La vida universitaria es una etapa en la que el ser humano se enfrenta a condiciones de gran demanda externa e interna, relacionadas al desarrollo profesional y personal, sobre todo orientado al futuro desempeño de un rol en la sociedad, lo cual le permitirá autosostenerse. El alumno universitario se encuentra en una etapa de vida impactante, ya que explora diversas posibilidades y comienza a formar su identidad basándose en el resultado de esas exploraciones. En ese contexto, surge el estrés como aquella resistencia a la tensión física, mental o emocional ocasionada por presiones y demandas ambientales, situacionales o personales, en que los sucesos impredecibles e incontrolables ocasionan más estrés que los hechos sobre los cuales los individuos tienen un mayor control (Tejeda et al., 2002). Esta temática resulta importante porque el estrés puede actuar como mediador entre las situaciones agobiantes (Sandin y Chorot, 2003). El estrés interviene en todos los ámbitos en los que se desenvuelve la persona y ejerce un impacto psicofisiológico perjudicial por desempeñar un papel desencadenador, regulador o exacerbador de diferentes síntomas (problemas para respirar con facilidad, taquicardia, insomnio, gastritis, colitis, dermatitis, migraña, etc.), así como estados emocionales perjudiciales (poca tolerancia a la frustración, ansiedad, depresión, temor, etc.), que son señalados por Pérez (2002, como se citó en Serrano y Flores, 2005).

Tomando a Frydenberg (1997), quien refiere que nuestras acciones frente al estrés y los recursos que tenemos para manejarlos determinan, en gran parte, el proceso de aprendizaje y de desarrollo del individuo, así como también su calidad de vida, se plantea la presencia de modos o estilos de afrontamiento frente a situaciones de estrés. Los modos de afrontamiento del estrés fueron analizados por Carver, Scheir y Weintraub (1989) tomando como base el modelo teórico de Lazarus y Folkman (1984) y los trabajos sobre evaluación del afrontamiento de Moos y Billings (1982); se tomó la clasificación de las dimensiones generales del afrontamiento (afrontamiento centrado en la evaluación, afrontamiento centrado en el problema y afrontamiento centrado en la emoción). Lazarus y Folkman (1988) manejan dos tipos de afrontamiento dentro de su teoría: a) el afrontamiento orientado al problema, se relaciona con el cambio de la problemática de la relación persona-ambiente mediante la actuación sobre el medio o sobre uno mismo; y b) el afrontamiento orientado a la emoción, que significa modificar el modo en que la relación estresante con el medio ambiente se lleva a cabo o el significado de lo que está sucediendo con el encuentro estresante, el cual mitiga el estado de tensión.

Carver, Scheir y Weintraub (1989), por su parte, proponen trece dimensiones que se agrupan en las dos categorías clásicas de Lazarus. Las estrategias centradas en el problema estarían expresadas por el afrontamiento activo, la planificación, la supresión de actividades competentes, la postergación del afrontamiento, la búsqueda de apoyo social por razones instrumentales; las estrategias 
centradas en la emoción se agrupan en las siguientes dimensiones: búsqueda de apoyo social por razones emocionales, reinterpretación positiva y crecimiento, aceptación, negación, acudir a la religión. Los citados autores encontraron que tres estrategias de afrontamiento no se agrupaban en las dos categorías anteriores y las unieron en una tercera llamada otras escalas, las cuales hacen referencia a estrategias usualmente consideradas inadecuadas para manejar el estrés; enfocar y liberar emociones, desentendimiento conductual y desentendimiento mental.

Los estudiantes se encuentran expuestos a situaciones de demanda, las que les permitirán consolidar sus propias formas de afrontamiento del estrés, que pueden o no ser exitosas; por ello, los repertorios conductuales de asertividad y los sentimientos de bienestar subjetivo son importantes. Las competencias asertivas permiten un adecuado manejo interpersonal de situaciones sociales, y los sentimientos de bienestar aportan la dosis de tranquilidad y optimismo suficientes para desarrollar estrategias de afrontamiento de los problemas cotidianos (Velasquez et al., 2008).

Ahora bien, el bienestar psicológico para Taylor (1991, como se citó en Leal y Silva, 2010) es parte de la salud en su sentido más general y se manifiesta en todas las esferas de la actividad humana. A decir del citado autor, cuando un individuo se siente bien, es más productivo, sociable y creativo; posee una proyección a futuro positiva; infunde felicidad, y esta implica capacidad de amar, trabajar, relacionarse socialmente y controlar el medio. Por su parte, Ryff y Keyes (1995) caracterizan el bienestar como la lucha por la perfección que representa la realización del propio potencial. Para estos autores, la felicidad corresponde a un resultado derivado de "la vida bien vivida". Y esta última debe reflejar los ideales de autonomía, crecimiento personal, autoaceptación, propósito en la vida, dominio del ambiente y relaciones positivas con los otros.

La variable bienestar psicológico ha centrado su atención en el desarrollo de las capacidades y el crecimiento personal, concebidas ambas como los principales indicadores del funcionamiento positivo. Sus primeras concepciones se articularon en torno a conceptos como la autoactualización (Maslow, 1968, como se citó en Leal y Silva, 2010), el funcionamiento pleno (Rogers, 1961, como se citó en Leal y Silva, 2010). A pesar de su relevancia teórica, ninguna de ellas llegó a tener un impacto significativo en los estudios de bienestar, debido, fundamentalmente, a la ausencia de fiabilidad y validez en los procedimientos de medida. En la búsqueda de puntos de convergencia entre todas estas formulaciones, Ryff (1989) sugiere un modelo multidimensional de bienestar psicológico compuesto por seis dimensiones: autoaceptación, relaciones positivas con otras personas, autonomía, dominio del entorno, propósito en la vida y crecimiento personal.

En el extranjero, se han realizado una serie de investigaciones sobre los modos de afrontamiento del estrés. Gonzales et al. (2002), en su investigación desarrollada 
en España, estudiaron la relación entre el afrontamiento y el bienestar psicológico, y las mujeres poseyeron un mayor repertorio de estrategias de afrontamiento con respecto a los hombres. Serrano y Flores (2005), en México, analizaron la relación entre el afrontamiento del estrés y la ideación suicida; ellos concluyeron que los hombres tenían más ideación suicida por el estrés en la relación de pareja, mientras que en las mujeres era mayor el estrés social (conflictos con amistades), y el estrés de pareja ocupaba un segundo lugar. Contreras, Chávez y Aragon (2012), en México, estudiaron la relación entre el pensamiento constructivo y el afrontamiento del estrés en estudiantes universitarios; los autores establecieron una relación fuerte entre ambas variables de estudio, y es el afrontamiento conductual el que más fuerte asociación presenta con el afrontamiento centrado en el problema. A nivel nacional, Boullosa (2013) investigó la relación entre el estrés y el afrontamiento en estudiantes de una universidad privada de Lima; la autora determinó que los estresores más frecuentes fueron la sobrecarga de trabajos y las evaluaciones de los profesores; además, la habilidad asertiva e implementación de un plan fueron las estrategias de afrontamiento más frecuentemente usadas por los estudiantes universitarios.

En relación con la variable bienestar psicológico, estudios internacionales como el de Loreto y Cumsille (1996), en Chile, determinaron las relaciones entre los niveles percibidos de apoyo social de la familia y de los amigos, los patrones de competencia psicosocial y la calidad de la utilización del tiempo libre como predictores del bienestar psicológico en adolescentes chilenos. En Argentina, Benatuil (2004) realizó un estudio cualitativo sobre la autopercepción del bienestar psicológico; determinó que las dimensiones vínculos personales y aceptación de sí mismo discriminan a los jóvenes con alto y bajo bienestar psicológico. En México, Chávez (2006) encontró una fuerte relación entre el bienestar psicológico y el rendimiento académico en estudiantes de bachillerato de la Universidad de Colima. Gómez y Cogollo (2010), en Colombia, tuvieron el objetivo de determinar los factores predictores relacionados con bienestar general en adolescentes estudiantes de Cartagena; los autores determinaron que los factores predictores fueron la alta autoestima, alta religiosidad y estar en una familia funcional. Castella et al. (2012) compararon los niveles de bienestar subjetivo de los adolescentes brasileros y argentinos, y encontraron diferencias a favor de los adolescentes de Argentina. A nivel nacional, Meza (2011) describe el bienestar psicológico de un grupo de practicantes de danza contemporánea; determinó que las mujeres tienen un mejor bienestar psicológico, particularmente en la dimensión meta de vida. $\mathrm{Y}$ aquellos que practican más horas a la semana la danza contemporánea poseen mayor bienestar psicológico.

Sobre la base de lo expuesto, se hace necesario conocer cuáles son los estilos de afrontamiento del estrés, así como identificar el nivel de bienestar psicológico de los estudiantes universitarios. Ambos podrían marcar su desempeño 
como estudiantes y futuros profesionales no solo como un logro bajo las demandas sociales, sino personales, con las cuales se puedan sentir gratificados y satisfechos. La presente investigación aborda la relación entre el afrontamien-to del estrés y el bienestar psicológico en estudiantes universitarios de dos localidades de Perú.

\section{Objetivos}

\section{Objetivo general}

Determinar la relación entre afrontamiento del estrés y bienestar psicológico en los estudiantes universitarios de Lima y Huancayo.

\section{Objetivos específicos}

1) Describir los niveles de los modos de afrontamiento del estrés en estudiantes universitarios de Lima y Huancayo

2) Describir los niveles del bienestar psicológico en estudiantes universitarios de Lima y Huancayo

3) Comparar el afrontamiento del estrés de los estudiantes universitarios en función de variables como el género, la ubicación geográfica y edad cronológica

4) Comparar el bienestar psicológico de los estudiantes universitarios en función de variables como el género, ubicación geográfica y edad cronológica

\section{Materiales y método}

En concordancia con las hipótesis formuladas, se empleó un tipo de investigación sustantiva-descriptiva, con análisis descriptivo, comparativo correlacional (Sánchez y Reyes, 2009). El diseño de investigación utilizado en el presente estudio fue no experimental, transeccional-correlacional.

La muestra estuvo constituida por 934 estudiantes cuyas edades fluctuaban entre los 16 y 24 años, procedentes de universidades públicas $(\mathrm{n}=535)$ y privadas $(\mathrm{n}=399)$ de Lima y Huancayo, conformada tanto por varones $(n=390)$ como por mujeres $(n=544)$. Dicha muestra fue seleccionada mediante muestreo aleatorio simple. Para efectos de los análisis comparativos según edad, se consideró como adolescentes a los estudiantes cuyas edades fluctaban entre los 16 y 20 años, y adultos jóvenes a los estudiantes entre 21 y 25 años. Dentro del estudio, se utilizó el consentimiento informado para los mayores de 18 años, y se usaron tanto el consentimiento como el asentimiento informado en el caso de los menores de edad.

\section{Técnica e instrumentos}

Dado el carácter de los fenómenos a estudiar, se utilizaron la técnica psicométrica y de observación indirecta. Los instrumentos utilizados fueron los siguientes:

La Escala de Modos de Afrontamiento al Estrés (COPE) fue diseñada por Carver, Scheir y Weintraub (1989), y adaptada a Perú por Casuso (1996). Tiene por objeto conocer las diferencias emocionales, cognitivas y conductuales que despliegan las personas al enfrentarse a diversas situaciones estresantes. Está 
compuesta por 52 ítems dicotómicos. Su administración es individual y colectiva, y es aplicable a sujetos de 16 años a más, con una duración de 20 minutos aproximadamente. Se adaptó este instrumento parael presente estudio; así, para la confiabilidad de consistencia interna del COPE, se utilizó el coeficiente Kuder-Richardson 20 (KR-20) al tratarse de una prueba con respuesta dicotómica (Kerlinger y Lee, 2002). Se obtuvo un coeficiente KR-20 de .733 , nivel considerado moderado (Murphy y Davishofer, 1998). En el caso de las dimensiones, poseían un KR-20 entre .673 a .626, ubicado en el nivel bajo, pero aceptable (Murphy y Davishofer, 1998).

La Escala de Bienestar Psicológico (SPWB), propuesta por Carol Ryff (1989), que es de administración individual y colectiva en un tiempo aproximado de 10 a 15 minutos, y está dirigida a adolescentes y jóvenes desde los 16 años en adelante. La escala adaptada contiene 32 ítems, distribuidos también en 6 dimensiones: autoaceptación (6 ítems), relaciones positivas (5 ítems), autonomía (5 ítems), dominio del entorno (6 ítems), propósito en la vida (5 ítems) y crecimiento personal (5 ítems). Las respuestas están formuladas según el modelo Likert y las puntuaciones son de cuatro puntos, dependiendo de las respuestas de los evaluados: siempre, casi siempre, casi nunca y nunca. La confiabilidad se hizo mediante un análisis de consistencia interna alfa de Cronbach; se obtuvo un coeficiente significativamente aceptable para la exploración del bienestar psicológico en los estudiantes: .88. La evaluación de la validez fue a través de las correlacio- nes entre las dimensiones y el test. Se observaron las correlaciones entre bienestar psicológico y las dimensiones que la componen, que asumieron, en el caso de autoaceptación, un valor de .806; en el caso de relaciones positivas, un valor de .701; en el caso de autonomía, un valor de .660; en el caso de dominio del entorno, un valor de .720; en el caso de crecimiento personal, un valor de .727, y en el caso de propósito en la vida, un valor de .776 . Estas correlaciones fueron estadísticamente significativas y positivas.

\section{Procesamiento de resultados}

Los datos se analizaron a través del programa estadístico SPSS versión 21. Los datos descriptivos, frecuencias y porcentajes se aplicaron para las variables del estudio. para el nivel del análisis inferencial, se utilizó previamente la prueba de normalidad de Kolmogorov-Smirnov y se determinó el uso de pruebas paramétricas. Para evaluar el tamaño del efecto de las correlaciones, se usó la $d$ de Cohen. Finalmente, la confiabilidad de los instrumentos se analizó con el alfa de Cronbach.

\section{Resultados}

\section{Análisis descriptivo}

La Tabla 1 presenta la variable modos de afrontamiento y sus dimensiones en los estudiantes universitarios de Lima y Huancayo. En cuanto a variable y dimensión, alcanzaron niveles predominantemente medios tanto en estudiantes universitarios de Lima como de Huancayo. 
En la tabla 2, se aprecia que la variable Bienestar Psicológico y sus dimensiones, igualmente, lograron niveles predominantemente medios en estudiantes universitarios de Lima y Huancayo.

\section{Tabla 1}

Resultados descriptivos de la variable modos de afrontamiento y sus dimensiones en estudiantes universitarios de Lima y Huancayo ( $n=934)$

\begin{tabular}{lccccc}
\hline & & \multicolumn{2}{c}{ Lima } & \multicolumn{2}{c}{ Huancayo } \\
\cline { 3 - 6 } & & $\mathrm{f}$ & $\%$ & $\mathrm{f}$ & $\%$ \\
\cline { 3 - 6 } & Alta & 63 & 11.8 & 47 & 11.8 \\
Modos de afrontamiento & Media & 410 & 76.8 & 319 & 79.8 \\
& Baja & 61 & 11.4 & 34 & 8.5 \\
& Alta & 62 & 11.6 & 62 & 11.6 \\
Estilos de afrontamiento & Media & 399 & 74.7 & 399 & 74.7 \\
centrado en el problema & Baja & 73 & 13.7 & 73 & 13.7 \\
& Alta & 51 & 9.6 & 45 & 11.3 \\
Estilos de afrontamiento & Media & 399 & 74.7 & 306 & 76.5 \\
centrado en la emoción & Baja & 84 & 15.7 & 49 & 12.3 \\
& Alta & 77 & 14.4 & 56 & 14.0 \\
Otros estilos de afrontamiento & Media & 371 & 69.5 & 295 & 73.8 \\
& Baja & 86 & 16.1 & 49 & 12.3 \\
Total & & 534 & 100.0 & 400 & 100.0 \\
\hline
\end{tabular}

\section{Tabla 2}

Resultados descriptivos de la variable bienestar psicológico y sus dimensiones en estudiantes universitarios de Lima y Huancayo $(n=934)$

\begin{tabular}{lccccc}
\hline & & \multicolumn{2}{c}{ Lima } & \multicolumn{2}{c}{ Huancayo } \\
\cline { 3 - 6 } & & $\mathrm{f}$ & $\%$ & $\mathrm{f}$ & $\%$ \\
\hline Bienestar psicológico & Alta & 89 & 16.7 & 51 & 12.8 \\
& Media & 395 & 74.0 & 302 & 75.5 \\
\multirow{4}{*}{ Autoaceptación } & Baja & 50 & 9.4 & 47 & 11.8 \\
& Alta & 69 & 12.9 & 50 & 12.5 \\
\multirow{3}{*}{ Relaciones positivas } & Media & 435 & 81.5 & 299 & 74.8 \\
& Baja & 70 & 13.1 & 51 & 12.8 \\
\multirow{3}{*}{ Autonomía } & Alta & 85 & 15.9 & 51 & 12.8 \\
& Media & 386 & 72.3 & 293 & 73.3 \\
& Baja & 63 & 11.8 & 56 & 14.0 \\
& Alta & 69 & 12.9 & 43 & 10.8 \\
& Media & 405 & 75.8 & 299 & 74.8 \\
& Baja & 60 & 11.2 & 58 & 14.5 \\
\hline
\end{tabular}




\begin{tabular}{|c|c|c|c|c|c|c|}
\hline & & & \multicolumn{2}{|c|}{ Lima } & \multicolumn{2}{|c|}{ Huancayo } \\
\hline & & & $f$ & $\%$ & $\mathrm{f}$ & $\%$ \\
\hline \multirow[t]{3}{*}{ Dominio del entorno } & Alta & & 84 & 15.7 & 51 & 12.8 \\
\hline & Media & & 399 & 74.7 & 304 & 76.0 \\
\hline & Baja & & 51 & 9.6 & 45 & 11.3 \\
\hline \multirow[t]{3}{*}{ Crecimiento personal } & Alta & & 73 & 13.7 & 36 & 9.0 \\
\hline & Media & & 402 & 75.3 & 303 & 75.8 \\
\hline & Baja & & 59 & 11.0 & 61 & 15.3 \\
\hline \multirow[t]{4}{*}{ Propósito en la vida } & Alta & & 58 & 10.9 & 41 & 10.3 \\
\hline & Media & & 414 & 77.5 & 321 & 80.3 \\
\hline & Baja & & 62 & 11.6 & 38 & 9.5 \\
\hline & & Total & 534 & 100.0 & 400 & 100.0 \\
\hline
\end{tabular}

\section{ANÁLISIS COMPARATIVO}

Se observa, en la Tabla 3, que la variable modos de afrontamiento alcanzó una media aritmética de 82.97 y una dispersión de 5.985. Por otro lado, respecto a la variable bienestar psicológico, se obtuvo un promedio de 172.66 , y una variabilidad de 24.281.

En la misma Tabla 3, se aplicó la prueba de Normalidad $\mathrm{Z}$ de Kolmogorov-Smirnov, con la que se determinó que la variable modos de afrontamiento y sus dimensiones alcanzan un $\mathrm{p}$-valor inferior al .01; es decir, tienen una distribución normal. Por otro lado, la variable bienestar psicológico y su dimensión autonomía alcanzaron un $\mathrm{p}$-valor superior al .05 , lo que indica que no poseen una distribución normal; en contraste, el resto de dimensiones de la variable bienestar psicológico alcanzó un p-valor inferior al .01 , indicativo de que dichas dimensiones poseen distribución normal.

\section{Tabla 3}

Estadísticos y prueba de Normalidad de las variables (modos de afrontamiento y bienestar psicológico)

\begin{tabular}{lcccc}
\hline Variable y dimensiones & $M$ & D. E. & K-S Z & $p$ \\
\hline $\begin{array}{l}\text { Modos de afrontamiento } \\
\begin{array}{l}\text { Estilos de afrontamiento } \\
\text { centrado en el problema }\end{array}\end{array}$ & 82.97 & 5.90 & 1.80 & .003 \\
$\begin{array}{l}\text { Estilos de afrontamiento } \\
\text { centrado en la emoción }\end{array}$ & 34.07 & 3.19 & 3.45 & $<.001$ \\
$\begin{array}{l}\text { Otros estilos de } \\
\text { afrontamiento }\end{array}$ & 17.04 & 3.06 & 2.84 & $<.001$ \\
\hline
\end{tabular}




\begin{tabular}{lcccc} 
(continuación) & \multicolumn{5}{c}{} \\
\hline Variable y dimensiones & $M$ & D. E. & K-S Z & $p$ \\
\hline Bienestar psicológico & 172.66 & 24.28 & 1.21 & .100 \\
Autoaceptación & 26.27 & 5.12 & 2.46 & $<.001$ \\
Relaciones positivas & 25.22 & 5.79 & 2.27 & $<.001$ \\
Autonomía & 33.59 & 6.38 & 1.23 & .098 \\
Dominio del entorno & 26.67 & 4.77 & 1.92 & .001 \\
Crecimiento personal & 32.66 & 5.17 & 2.21 & $<.001$ \\
Propósito en la vida & 28.26 & 5.41 & 2.97 & $<.001$ \\
\hline
\end{tabular}

En el análisis comparativo, se utilizó la prueba paramétrica $t$ de Student para muestras independientes para la variable bienestar psicológico y sus dimensiones, que poseían una distribución normal (véase tabla 3). En la tabla 4, se aprecia que, únicamente en la dimensión estilos de afrontamiento centrado en la emoción, hubo diferencias significativas $(p<.01)$ entre ambos grupos (Lima y Huancayo) a favor del grupo de Huancayo. En contraste, en la variable Modos de Afrontamiento y las dimensiones Estilos de afrontamiento centrado en el problema y Otros estilos de afrontamiento, no hubo diferencias significativas entre ambos grupos.

\section{Tabla 4}

Prueba t de Student para muestras independientes para estimar si existen diferencias en la variable modos de afrontamiento y sus dimensiones según ubicación geográfica $(n=934)$

\begin{tabular}{lccccc}
\hline Variable y dimensiones & Grupo & $n$ & Media & $t$ & $d_{\text {Cohen }}$ \\
\hline \multirow{2}{*}{ Modos de afrontamiento } & Lima & 534 & 82.75 & -1.318 & 0.09 \\
& Huancayo & 400 & 83.26 & & \\
Estilos de afrontamiento & Lima & 534 & 34.17 & 1.113 & 0.08 \\
centrado en el problema & Huancayo & 400 & 33.93 & & \\
Estilos de afrontamiento & Lima & 534 & 31.59 & $-3.207^{* *}$ & 0.29 \\
centrado en la emoción & Huancayo & 400 & 32.24 & & \\
Otros estilos de & Lima & 534 & 16.99 & -.714 & 0.01 \\
afrontamiento & Huancayo & 400 & 17.10 & & \\
\hline
\end{tabular}

Nota: ${ }^{* *} p<.01$ 
Por otra parte, con la misma prueba paramétrica $t$ de Student para muestras independientes, se analizaron las dimensiones de bienestar psicológico, autoaceptación, relaciones positivas, dominio del entorno, crecimiento personal y propósito en la vida, al poseer todas ellas una distribución normal (véase tabla 3).
En la Tabla 5, la dimensión crecimiento personal logró diferencias significativas $(p<.01)$ entre ambos grupos (Lima y Huancayo) a favor del grupo de Lima. Sin embargo, en el resto de dimensiones no se encontró diferencias significativas entre ambos grupos.

\section{Tabla 5}

Prueba t de Student para muestras independientes para estimar si existen diferencias en las dimensiones de la variable bienestar psicológico según ubicación geográfica

\begin{tabular}{lccccc}
\hline Variable y dimensiones & Grupo & $n$ & Media & $t$ & $d_{\text {Cohen }}$ \\
\hline \multirow{2}{*}{ Autoaceptación } & Lima & 534 & 26.36 & .620 & 0.04 \\
& Huancayo & 400 & 26.15 & & 0.12 \\
Relaciones positivas & Lima & 534 & 25.53 & 1.875 & \\
& Huancayo & 400 & 24.80 & & 0.14 \\
Dominio del entorno & Lima & 534 & 26.90 & 1.705 & \multirow{2}{*}{0.29} \\
& Huancayo & 400 & 26.36 & & \\
Crecimiento personal & Lima & 534 & 33.18 & $3.582^{* *}$ & 0.01 \\
& Huancayo & 400 & 31.96 & & \\
Propósito en la vida & Lima & 534 & 28.26 & -.006 & \\
& Huancayo & 400 & 28.26 & & \\
\hline
\end{tabular}

Nota: ${ }^{* *} p<.01$

Tabla 6

Prueba no paramétrica $U$ de Mann-Whitney para muestras independientes en la variable bienestar psicológico y la dimensión autonomía según ubicación geográfica $(n=934)$

\begin{tabular}{|c|c|c|c|c|c|c|}
\hline Variable y Dimensión & Grupo & $n$ & Media & $U$ & $Z$ &.$r$ \\
\hline \multirow{2}{*}{ Bienestar psicológico } & Lima & 534 & 485.16 & \multirow{2}{*}{97368.00} & \multirow{2}{*}{-2.31} & \multirow{2}{*}{.08} \\
\hline & Huancayo & 400 & 443.92 & & & \\
\hline \multirow{2}{*}{ Autonomía } & Lima & 534 & 491.85 & \multirow{2}{*}{93796.50} & \multirow{2}{*}{-3.19} & \multirow{2}{*}{.10} \\
\hline & Huancayo & 400 & 434.99 & & & \\
\hline
\end{tabular}

Nota $=r$ es usado como estimación del tamaño del efecto

${ }^{*} p<.02{ }^{* *} p<.01$ 
En la tabla 6, se obtuvo las comparaciones de la variable bienestar psicológico y la dimensión autonomía través de la prueba no paramétrica U de Mann-Whitney, al no poseer ambas una distribución normal (véase tabla 3). Se encontró diferencias en la variable bienestar psicológico $(p<.05)$ y su dimensión autonomía $(p<.01)$ a favor de los estudiantes de Lima.

En la Tabla 7, se aprecia que, en las dimensiones estilos de afrontamiento centrado en el problema y otros estilos de afrontamiento, hay diferencias significativas $(p<.05)$ entre ambos grupos (hombre y mujer) a favor del grupo de mujeres en ambos casos. En contraste, en la variable modos de afrontamiento y la dimensión
Estilos de afrontamiento centrado en la emoción, no hubo diferencias significativas entre ambos grupos.

Por otra parte, en la Tabla 8, se detectó, en las dimensiones crecimiento personal y propósito en la vida, diferencias significativas $(p<.01)$ entre ambos grupos (hombre y mujer) a favor del grupo de mujeres en ambos casos. Sin embargo, en el resto de dimensiones, no se encontró diferencias significativas entre ambos grupos.

Se aprecia, en la Tabla 9, diferencias significativas en la variable bienestar psicológico $(p<.01)$ a favor del grupo de mujeres. Sin embargo, no se encontró diferencias significativas en la dimensión autonomía.

\section{Tabla 7}

Prueba t de Student para muestras independientes para estimar si existen diferencias en la variable modos de afrontamiento y sus dimensiones según género $(n=934)$

\begin{tabular}{lccccc}
\hline Variable y dimensiones & Grupo & $n$ & Media & $t$ & dCohen \\
\hline \multirow{2}{*}{ Modos de afrontamiento } & Hombre & 390 & 81.72 & -5.567 & 0.36 \\
& Mujer & 544 & 83.86 & & \\
Estilos de afrontamiento centrado & Hombre & 390 & 33.77 & $-2.407^{*}$ & 0.16 \\
en el problema & Mujer & 544 & 34.28 & & \\
Estilos de afrontamiento centrado & Hombre & 390 & 31.10 & -6.620 & 0.43 \\
en la emoción & Mujer & 544 & 32.42 & & \\
Otros estilos de afrontamiento & Hombre & 390 & 16.85 & $-2.100^{*}$ & 0.14 \\
& Mujer & 544 & 17.17 & & \\
\hline
\end{tabular}

Nota: ${ }^{*} p<.05$ 
Tabla 8

Prueba t de Student para muestras independientes para estimar si existen diferencias en las dimensiones de la variable bienestar psicológico según género $(n=934)$

\begin{tabular}{lccccc}
\hline \multicolumn{1}{c}{ Variable y dimensiones } & Grupo & $n$ & Media & $t$ & $d_{\text {Cohen }}$ \\
\hline \multirow{2}{*}{ Autoaceptación } & Hombre & 390 & 26.18 & -.426 & 0.03 \\
& Mujer & 544 & 26.33 & & \\
Relaciones positivas & Hombre & 390 & 24.81 & -1.839 & 0.13 \\
& Mujer & 544 & 25.51 & & \\
Dominio del entorno & Hombre & 390 & 26.38 & -1.550 & 0.11 \\
& Mujer & 544 & 26.88 & & \multirow{2}{*}{0.24} \\
Crecimiento personal & Hombre & 390 & 31.95 & $-3.600^{* *}$ & \\
& Mujer & 544 & 33.17 & & 0.25 \\
Propósito en la vida & Hombre & 390 & 27.52 & $-3.575^{* *}$ & \\
\hline
\end{tabular}

Nota: ${ }^{* *} p<.01$

Tabla 9

Prueba no paramétrica $U$ de Mann-Whitney para muestras independientes en la variable bienestar psicológico y la dimensión autonomía según género $(n=934)$

\begin{tabular}{lcccccc}
\hline \multicolumn{1}{c}{$\begin{array}{c}\text { Variable y } \\
\text { Dimensión }\end{array}$} & Grupo & $n$ & Media & $\begin{array}{c}\text { Ude Mann- } \\
\text { Whitney }\end{array}$ & Z & $r r$ \\
\hline Bienestar & Hombre & 390 & 442,08 & 96165.500 & $-2.439^{*}$ & .08 \\
psicológico & Mujer & 544 & 485,73 & & & \\
& Hombre & 390 & 478,77 & 101684.500 & -1.082 & .04 \\
\hline
\end{tabular}

Nota: $=$ r es usado como estimación del tamaño del efecto ${ }^{*} p<.05$

Se tiene, en la Tabla 10, que tanto la variable modos de afrontamiento como sus dimensiones no obtuvieron diferencias significativas entre los grupos de adolescentes y adultos jóvenes.

Por otro lado, la tabla 11 muestra que, en la variable bienestar psicológico, las dimensiones relaciones positivas, dominio de entorno y crecimiento personal poseen diferencias significativas entre ambos grupos (adolescentes y adultos jóvenes) a favor de los adultos jóvenes. Sin embargo, en el resto de dimensiones, no se encontró diferencias significativas entre ambos grupos. 
Tabla 10

Prueba t de Student para muestras independientes para estimar si existen diferencias en la variable modos de afrontamiento y sus dimensiones según edad cronológica $(n=934)$

\begin{tabular}{llcccc}
\hline \multicolumn{1}{c}{ Variable y dimensiones } & Grupo & $n$ & Media & $t$ & $d_{\text {cohen }}$ \\
\hline \multirow{2}{*}{ Modos de afrontamiento } & Adolescente & 476 & 82.92 & -.247 & 0.02 \\
& Adulto joven & 458 & 83.02 & & \\
Estilos de afrontamiento & Adolescente & 476 & 33.89 & -1.705 & 0.11 \\
centrado en el problema & Adulto joven & 458 & 34.25 & & \\
Estilos de afrontamiento & Adolescente & 476 & 31.91 & \multirow{2}{*}{0.13} \\
centrado en la emoción & Adulto joven & 458 & 31.83 & & \\
Otros estilos de & Adolescente & 476 & 17.12 & \multirow{2}{*}{1.171} & 0.08 \\
afrontamiento & Adulto joven & 476 & 82.92 & & \\
\hline
\end{tabular}

Tabla 11

Prueba t de Student para muestras independientes para estimar si existen diferencias en las dimensiones de la variable bienestar psicológico según edad cronológica $(n=934)$

\begin{tabular}{lccccc}
\hline Variable y dimensiones & Grupo & $n$ & Media & $t$ & $d_{\text {cohen }}$ \\
\hline \multirow{2}{*}{ Autoaceptación } & Adolescente & 476 & 25.99 & -1.669 & 0.11 \\
& Adulto joven & 458 & 26.55 & & \\
\multirow{2}{*}{ Relaciones positivas } & Adolescente & 476 & 24.64 & $-3.139^{* *}$ & 0.21 \\
& Adulto joven & 458 & 25.82 & & \\
Dominio del entorno & Adolescente & 476 & 26.26 & \multirow{2}{*}{0.17} \\
& Adulto joven & 458 & 27.09 & & \\
Crecimiento personal & Adolescente & 476 & 32.27 & $-2.368^{*}$ & 0.16 \\
& Adulto joven & 458 & 33.07 & & \\
Propósito en la vida & Adolescente & 476 & 28.31 & \multirow{2}{*}{.274} & 0.02 \\
& Adulto joven & 458 & 28.21 & & \\
\hline
\end{tabular}

Nota: ${ }^{* *} p<.01 ;{ }^{*} p<.05$ 


\section{Tabla 12}

Prueba no paramétrica $U$ de Mann-Whitney para muestras independientes en la variable bienestar psicológico y la dimensión autonomía según edad cronológica $(n=934)$

\begin{tabular}{lcccccc}
\hline \multicolumn{1}{c}{$\begin{array}{c}\text { Variable y } \\
\text { Dimensión }\end{array}$} & Grupo & $n$ & Media & $\begin{array}{c}\text { Ude Mann- } \\
\text { Whitney }\end{array}$ & Z & $r$ \\
\hline Bienestar & Adolescente & 476 & 438.45 & 95177.000 & $-3.36^{* *}$ & .11 \\
psicológico & Adulto joven & 458 & 497.69 & & & \\
& Adolescente & 476 & 438.39 & & \\
Autonomía & Adulto joven & 458 & 497.76 & 95146.000 & $-3.37^{* *}$ & .11 \\
& & & & &
\end{tabular}

Nota: $r$ es usado como estimación del tamaño del efecto ${ }^{* *} p<.01$

Se observa, en la Tabla 12, diferencias significativas en la variable bienestar psicológico $(p<.01)$ y su dimensión autonomía $(p<.01)$, a favor del grupo de adultos jóvenes en ambos casos.

\section{ANÁLISIS CORRELACIONAL}

Se realizó un análisis de la correlación mediante la prueba paramétrica $\mathrm{R}$ de Pearson al poseer un $p>.05$ (véase Tabla 3). Se observa, en la Tabla 13, una correlación directa entre la variable modos de afrontamiento y la dimensión propósito en la vida. Y en la dimensión estilos de afrontamiento centrado en el problema se halló correlaciones directas con todas las dimensiones del bienestar psicológico. Para el caso de la dimensión estilos de afrontamiento centradoenlaemoción, hay correlación directa con las dimensiones relaciones positivas y propósito en la vida. En contraste, la dimensión otros estilos de afrontamiento se relacionó de forma inversa con todas las dimensiones del bienestar psicológico.

Por otro lado, se realizó un análisis de la correlación con las variables y dimensiones que no poseían una distribución normal (véase Tabla 3), para lo cual se usó la prueba no paramétrica Rho de Spearman. En la tabla 14, se halló una correlación directa entre la variable modos de afrontamiento y la dimensión autonomía. De igual modo, entre la dimensión estilos de afrontamiento centrado en la emoción y la dimensión autonomía, hubo una correlación indirecta. La dimensión Otros estilos de afrontamiento se relacionó de forma indirecta con la variable bienestar psicológico y la dimensión autonomía. 
Tabla 13

Correlaciones $R$ de Pearson entre los modos de afrontamiento y las dimensiones de bienestar psicológico

\begin{tabular}{lccccc}
\hline \multirow{2}{*}{ Afrontamiento } & \multicolumn{5}{c}{ Dimensiones de Bienestar Psicológico } \\
\cline { 2 - 6 } & $\begin{array}{c}\text { Auto- } \\
\text { aceptación }\end{array}$ & $\begin{array}{c}\text { Relaciones } \\
\text { positivas }\end{array}$ & $\begin{array}{c}\text { Dominio } \\
\text { del entorno }\end{array}$ & $\begin{array}{c}\text { Crecimiento } \\
\text { personal }\end{array}$ & $\begin{array}{c}\text { Propósito } \\
\text { en la vida }\end{array}$ \\
Modos de afrontamiento & -.054 & .027 & .000 & .000 & $.089^{* *}$ \\
$\begin{array}{l}\text { Estilos de afrontamiento } \\
\text { centrado en el problema }\end{array}$ & $.089^{* *}$ & $.099^{* *}$ & $.162^{* *}$ & $.131^{* *}$ & $.205^{* *}$ \\
$\begin{array}{l}\text { Estilos de afrontamiento } \\
\text { centrado en la emoción }\end{array}$ & .014 & $.083^{*}$ & .030 & .000 & $.120^{* *}$ \\
$\begin{array}{l}\text { Otros estilos de } \\
\text { afrontamiento }\end{array}$ & $-.281^{* *}$ & $-.178^{* *}$ & $-.266^{* *}$ & $-.181^{* *}$ & $-.218^{* *}$ \\
\hline
\end{tabular}

Nota: ${ }^{* *} \mathrm{p}<.01$

\section{Tabla 74}

Correlaciones Rho de Spearman entre los modos de afrontamiento y el bienestar psicológico y su dimensión autonomía

\begin{tabular}{lcc}
\hline Afrontamiento & $\begin{array}{c}\text { Bienestar } \\
\text { psicológico }\end{array}$ & Autonomía \\
\hline Modos de afrontamiento & -.025 & $-.126^{* *}$ \\
$\begin{array}{l}\text { Estilos de afrontamiento centrado en } \\
\text { el problema }\end{array}$ & $.118^{* *}$ & .034 \\
$\begin{array}{l}\text { Estilos de afrontamiento centrado en } \\
\text { la emoción }\end{array}$ & .024 & $-.096^{* *}$ \\
Otros estilos de afrontamiento & $-.292^{* *}$ & $-.238^{* *}$ \\
\hline
\end{tabular}

Nota: ${ }^{* *} p<.01$ 


\section{DISCUSIÓN DE RESULTADOS}

El presente estudio fue realizado en dos localidades del Perú, con 534 estudiantes de Lima y 400 de Huancayo. En el análisis descriptivo, se obtuvo que tanto para los estudiantes de Lima como para los de Huancayo los modos de afrontamiento y sus dimensiones se ubicaron en niveles predominantemente medios, lo cual implica que los estudiantes universitarios de ambas localidades adoptan estilos de afrontamiento centrados en los problemas, centrados en las emociones y otros etilos de afrontamiento en un nivel promedio. Es importante tomar en cuenta que esto depende de las interpretaciones que las personas hacen. Cada estrategia de afrontamiento puede producir resultados positivos en un contexto o en una persona en particular, pero no en otro. Lo que se sugiere es investigar las circunstancias particulares de estrategias de afrontamiento que producen resultados adaptativos o desadaptativos (Lazarus, 1993). Por otro lado, la variable bienestar psicológico y sus dimensiones, igualmente, lograron niveles predominantemente medios en estudiantes universitarios de Lima y Huancayo. Cabe considerar factores sociales y personales que podrían estar relacionados con la percepción de un nivel medio en el bienestar psicológico, como los hallados por Loreto y Cumsille (1996), quienes identificaron que la calidad del uso del tiempo libre, la competencia social y el apoyo social familiar se asocian de manera significativa con el bienestar psicológico de los adolescentes estudiados en Chile.
En comparación, en la dimensión de modos de afrontamiento centrados en la emoción, se obtuvo diferencias significativas entre ambos grupos poblacionales a favor de los estudiantes universitarios de la ciudad de Huancayo, lo cual denota que tienden a modificar el modo en que la relación estresante con el medio ambiente se lleva a cabo o el significado de lo que está sucediendo en el encuentro estresante, lo cual mitiga el estado de tensión. Esto lo podemos asociar a un estudio desarrollado por Cassaretto (2009) en adolescentes preuniversitarios del Perú, en el que obtuvo que el estilo centrado en las emociones se relaciona de forma significativa con calidez, emociones positivas y benevolencia; dichas características podrían ser rasgos de estudiantes residentes en un ciudad de 1 millón 331 000 habitantes (INEI, 2011), a diferencia de la ciudad de Lima, con una cantidad de 9 millones 541000 habitantes (INEI, 2011), donde el nivel de competitividad y estilo de vida varía significativamente.

En las dimensiones de crecimiento personal y autonomía de la variable bienestar psicológico, se obtuvo diferencias significativas a favor de los estudiantes de Lima; se entiende que en ellos predomina la necesitad de desarrollar sus potencialidades, de seguir creciendo como persona y llevar al máximo sus capacidades, así como la necesidad de asentarse en sus propias convicciones, y mantener su independencia y autoridad personal. Dichas diferencias guardarían correspondencia con factores sociodemográficos y con vivir en la ciudad capital, donde existe un alto nivel de exigencia y competitividad; 
en tal sentido, Triandis (1977) realizó estudios sobre la cultura y propone que el individualismo y colectivismo son características de las sociedades. Las culturas colectivistas que describe este investigador (mexicana) se caracterizan por la evitación de la competencia, y destaca en su lugar la cooperación, la ayuda mutua y la importancia de la familia. Por el contrario, en las culturas individualistas, las metas y logros personales están por encima de las metas del grupo; en ese sentido, el comportamiento de cada elemento de este binomio sociocultural es distinto, como distinta es su percepción y actuación en el mundo.

En cuanto a las diferencias según el género, en los modos de afrontamiento del estrés centrados en el problema y otros estilos de afrontamiento, se halló diferencias significativas a favor de las mujeres, lo que indica que estas buscan el cambio de la problemática de la relación persona-ambiente mediante la actuación sobre el medio o sobre ellas. En este sentido, Fierro (1996) y Lazarus y Folkman (1986) refieren que quienes adoptan modos de afrontamiento centrados en el problema mantienen un locus de control interno, ya que se consideran protagonistas de sus conductas, sienten que tienen control sobre el entorno cuando se presenta un evento estresante y que se pueden realizar acciones para suprimirlo. En el presente estudio, también se observó que las mujeres tienden a usar otros estilos de afrontamiento, los cuales hacen referencia a estrategias usualmente consideradas inadecuadas para manejar el estrés, a diferencia de los varones. Ambos resultados se condicen con estudios como los de González, Montoya, Casullo y Bernabéu (2002), quienes sostienen que las mujeres poseen mayor repertorio de estrategias de afrontamiento y menos habilidad para afrontar los problemas que los varones. Por otro lado, los resultados son similares a los obtenidos por Frydenberg y Lewis (1991), quienes señalan la existencia de diferencias de género en el estilo focalizado en la emoción, que estaría asociado a las mujeres.

En la variable bienestar psicológico se encontró que las mujeres perciben un mayor crecimiento personal y propósito en la vida, y en la escala general, perciben un mayor bienestar psicológico a diferentecia de los varones. Lo hallado refuerza los resultados del estudio de Leal y Silva (2010), quienes se interesaron por conocer el nivel y las características del bienestar psicológico en adolescentes de la ciudad de Cajamarca, y concluyeron que las mujeres presentan mayor bienestar psicológico que los estudiantes varones. Sin embargo, estos resultados difieren de los de un estudio a nivel internacional de Castella et al., (2012). Ellos presentaron una investigación, cuyo objetivo principal se enfocó en conocer y comparar los niveles de bienestar subjetivo de los adolescentes brasileros y argentinos, en la que los varones obtuvieron puntajes superiores que las mujeres. Dichas variaciones estarían asociadas a factores culturales; por ello, (Casullo y Castro, 2000) consideran que el género no parece afectar la percepción subjetiva del bienestar psicológico. 
Al efectuar comparaciones según sexo, se halló que los varones manifiestan mayor aceptación de sí mismos que las mujeres, lo que concuerda con lo señalado por Byrne (2000), quien afirma que las mujeres centrarían su bienestar en el control de las situaciones y en el establecimiento de vínculos psicosociales. La existencia de estas diferencias abre la indagación y refuerza que el género, posiblemente, no sea el único factor que afecte la percepción del bienestar psicológico.

Cuando se comparó según la edad, se encontró que, en la variable modos de afrontamiento, no hay diferencias significativas entre adolescentes y adultos jóvenes. Los resultados difieren de investigaciones consultadas, en las cuales se halló que el adolescente utiliza una jerarquía de estrategias para enfrentarse a los problemas específicos (Frydenberg y Lewis, 1994) y que con la edad aumenta su repertorio (Williams y McGillicudy, 2000). Por su parte, Sotelo y Maupone (2000) sugirieron que sí existen diferencias en los estilos de afrontamiento por tipos de muestras en cuanto a edad y cultura se refiere.

Del análisis anterior, se desprende la necesidad de realizar estudios que contemplen qué estilos de afrontamiento utilizan diferentes tipos de poblaciones, de diferentes edades y de diferentes culturas. Es probable que se necesite considerar un estudio a profundidad o se pueda hablar de una homogeneidad en los modos de afrontar el estrés en la población estudiada.

En la variable bienestar psicológico, se obtuvo que las dimensiones autono- mía, relaciones positivas, dominio del entorno, crecimiento personal y la escala general de bienestar psicológico se ven más desarrolladas en adultos jóvenes, lo cual contrasta con un estudio realizado por Robles, Sánchez y Galicia (2011), quienes encontraron una única diferencia por edad en la categoría de logros de objetivos a favor de los adolescentes de 18 y 19 años, debido a que la madurez y el crecimiento en esta etapa vital están determinados, en gran medida, por la toma de decisiones. La muestra del presente estudio oscila entre los 16 y 25 años, edades cruciales en la madurez mental y la autonomía.

En el análisis estadístico correlacional, se halló una relación significativa y directa entre los modos de afrontamiento del estrés y el bienestar psicológico, lo que significa que el modo de afrontamiento del estrés centrado en el problema guarda una relación directa con todas las dimensiones del Bienestar Psicológico.Esto implica que, ante un mayor uso de este modo de afrontamiento, caracterizado por el actuar sobre el problema, mayor será el bienestar psicológico que se experimente; lo corrobora el estudio de Boullosa (2013), quien identificó correlaciones significativas entre los estilos con la satisfacción con la vida y las estrategias de afrontamiento, así como el estudio realizado por Gonzales et al. (2002). Ellos investigaron en la ciudad de Valencia, España, la relación entre los estilos, estrategias de afrontamiento y bienestar psicológico en adolescentes; los resultados mostraron que había una escasa relación entre ambas variables, lo que especifica Cassaretto (2009) refiriendo 
que hay un mayor uso del estilo centrado en el problema. Es importante señalar que este es un estilo muy saludable para afrontar el estrés, ya que investigaciones previas establecen una fuerte relación con el bienestar físico, y se asocia con una mayor probabilidad de resolver los eventos. En la población universitaria, estas estrategias permiten una organización más eficiente a los alumnos; a través de ellas, pueden obtener los recursos que necesiten de los demás y favorece respuestas activas para enfrentar las dificultades. El modo de afrontamiento centrado en la emoción guarda una relación directa con las dimensiones de la variable bienestar psicológico de relaciones positivas y propósito en la vida, lo cual implica que aquellos que tratan de modificar el modo en que la relación estresante con el medio ambiente se lleva a cabo o el significado de lo que está sucediendo en el encuentro estresante tienden a mantener relaciones más positivas y propósitos de vida estables. Esto se sustenta en lo expuesto por Cassaretto (2009): el uso del estilo centrado en la emoción también es positivo, ya que es conocido que permite manejar emociones negativas e intensas, como la ansiedad y cólera. Lazarus y Lazarus (2000) señalan que este estilo es particularmente útil, ya que ayuda a controlar la angustia y disfunción que podría generarse cuando poco o nada puede hacerse en el momento para resolver definitivamente un evento, y es un estilo útil, ya que ayuda a mantener la moral en alto y el sentido de esperanza. Cabe mencionar que, en los resultados obtenidos, se resalta que el uso de otros estilos de afrontamiento (considerados como poco funcionales) registró una relación significativa e inversa con el bienestar psicológico y su dimensión de autonomía, lo cual se relaciona con lo hallado por Mikkelsen (2009), quien encontró que, en la medida que la satisfacción de los jóvenes disminuyó, hubo una preferencia por afrontar sus preocupaciones desde una posición pasiva, y se centraron en disminuir el malestar generado más que en buscar soluciones (afrontamiento no productivo), pues existe incapacidad para controlar y afrontar directamente. Así mismo, Cassaretto (2009) identificó que el uso de otros estilos (Alcalde, 1998; Casuso, 1996; Cassaretto et al., 2003), el alto uso de estrategias pasivas y paliativas, es el que genera mayor malestar físico y es considerado un estilo de afrontamiento de alto riesgo para desarrollar psicopatología (Seiffge-Krenke, 1998, 2000). El estudio de Cassaretto (2009) también obtuvo que los otros estilos de afrontamiento se relacionan de forma positiva con casi todas las escalas de neuroticismo (ansiedad, hostilidad colérica, depresión, impulsividad y vulnerabilidad); este estilo también se relaciona de forma negativa con la faceta de la autodisciplina.

A modo de conclusión, se puede señalar que el presente estudio valida la relación significativa entre los modos de afrontamiento del estrés y el bienestar psicológico, y considera que tanto un modo de afrontamiento centrado en el problema como un modo de afrontamiento centrado en la emoción generan predisposición a mantener el bienestar psicológico, a diferencia de otros modos de afrontamiento 
que son no adaptativos, que predisponen a una disminución o pérdida del bienestar psicológico. Por lo tanto, son datos relevantes a tomar en cuenta en futuros programas de intervención, así como en investigaciones con poblaciones universitarias, considerando que se encuentran en formación y es la etapa en que se constituyen y consolidan sus habilidades para el futuro desempeño en un rol social activo.

\section{Conclusiones}

1) En un nivel descriptivo, ambas poblaciones muestrales (Lima y Huancayo) mostraron un desempeño promedio en los modos de afrontamiento del estrés y el nivel de bienestar psicológico.

2) El modo de afrontamiento del estrés centrado en la emoción mantiene una diferencia significativa entre Lima y Huancayo a favor de los alumnos universitarios de Huancayo.

3) En la variable bienestar psicológico y sus dimensiones de autonomía y crecimiento personal, se obtuvo diferencias significativas entre Lima y Huancayo a favor de los estudiantes universitarios de Lima.

4) El modo de afrontamiento del estrés centrado en la emoción y otros estilos de afrontamiento guardan diferencias significativas entre varones y mujeres, a favor de las mujeres.

5) El bienestar psicológico y sus dimensiones de crecimiento personal y propósito en la vida guardan diferencias significativas entre varones y mujeres a favor de las mujeres.

6) El modo de afrontamiento del estrés y sus dimensiones no guardan diferencias significativas según la edad.

7) El bienestar psicológico y sus dimensiones de relaciones positivas, dominio del entorno, autonomía y crecimiento personal guardan diferencias significativas según la edad a favor de los adultos jóvenes.

8) En el análisis de correlaciones, se obtuvo una relación significativa y directa entre los modos de afrontamiento del estrés y el bienestar psicológico en los estudiantes universitarios; así mismo, los modos de afrontamiento del estrés centrados en el problema y la emoción mantienen una relación significativa directa con las dimensiones de bienestar psicológico, mientras que el uso de otros estilos de afrontamiento guardan una relación significativa e indirecta con el bienestar psicológico.

\section{RefERENCIAS}

Alcalde, M. (1998). Nivel de autoeficacia y estilos de afrontamiento en estudiantes universitarios de Lima (Tesis de maestría). Pontificia Universidad Católica del Perú, Lima-Perú.

Benatuil, D. (2004). El bienestar psicológico en adolescentes desde una perspectiva cualitativa. Psicodebate 3: Psicología, Cultura y Sociedad, 3, 43-58. 
Boullosa, G. (2013). Estrés académico y afrontamiento en un grupo de estudiantes de una universidad privada de Lima. Recuperado de http://tesis.pucp.edu.pe/ repositorio/handle/123456789/4880

Byrne, B. (2000). Relationships Between Anxiety, Fear, Self-Esteem and Coping Strategies in Adolescence. Adolescence, 55(37), 201-215.

Carver, C., y Scheier, M. (1997). Teorías de la personalidad. Mexico D.F.: Prentice Hall.

Carver, C., Scheier, M., y Weintraub, J. (1989). Assessing Coping Strategies: A Theoretically Based Approach.Journal of Personality and Social Psychology, 56(2), 267-283.

Castella, J., Saforcada, E., Tonon, G., Rodriguez, L., Mozobancyk S., y Bedin, M. (2012). Bienestar subjetivo de los adolescentes: estudio comparativo entre Argentina y Brasil. Psychosocial Intervention, 21(3), 273-280.

Cassaretto, M., Chau, C., Oblitas, H., y Valdés, N. (2003). Estrés y afrontamiento en estudiantes de psicología. Revista de Psicología de la Pontificia Universidad Católica del Perú, 21 (2), 363-392.

Casullo, M., y Castro, A. (2000). Evaluación del bienestar psicológico en estudiantes adolescentes argentinos. Revista de Psicología, 18(1), 36-38.

Casuso, L. (1996). Adaptación de la prueba COPE sobre estilos de afrontamiento en un grupo de estudiantes universitarios de Lima (Tesis de licen- ciatura inédita). Pontificia Universidad Católica del Perú, Lima-Perú.

Contreras, O., Chávez, M., y Aragón, L. (2012). Pensamiento constructivo y afrontamiento al estrés en estudiantes universitarios. Recuperado el 30 de noviembre de 2013 de http://132.247.146.34/index.php/jbhsi/article/view/32970

Chávez, A. (2006). Bienestar psicológico y su influencia en el rendimiento académico de estudiantes de nivel medio superior (Tesis de maestría). Universidad de Colima, Colima-México.

Fierro, A. (1996). Manual de Psicología de la Personalidad. Buenos Aires: Paidos.

Frydenberg, E. (1997). Adolescent Coping. Theoretical and Research Perspective. New York: Routjedge.

Frydenberg, E., y Lewis, R. (1994). Coping with Different Concerns: Consistency and Variation in Coping Strategies Used by Adolescents. Australian Psychologist, 29(1), 45-48.

Gómez, E., y Cogollo, Z. (2010). Factores predictores relacionados con el bienestar general en adolescentes estudiantes de Cartagena, Colombia. Revista de Salud Pública, 12(1), 61-70.

González, R., Montoya, I., Casullo, M., y Bernabéu, J. (2002). Relación entre estilos y estrategias de afrontamiento y bienestar psicológico en adolescentes. Psicothema 14(2), 363-368

Hernández, R., Fernández, C., y Baptista, P. (2010). Metodología de la investiga-

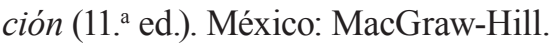


Lazarus, R. (1993). From Psychological Stress to the Emotions: a History of changing outlooks. Annual Review of Psychology, 44, 1-21.

Lazarus, R., y Folkman, S. (1986). Estrés y procesos cognitivos. Barcelona: Martínez Roca S.A.

Leal, R., y Silva, R. (2010). Bienestar psicológico del adolescente cajamarquino. Cajamarca: Universidad Privada Antonio Guillermo Urrello.

Loreto, P., y Cumsille, P. (1996) Bienestar Psicológico en adolescentes urbanos: su relación con niveles de competencia psicosocial, sistemas de apoyo social y calidad de tiempo libre. Revista Psyke, 5(2), 185-202.

Meza, B. (2010). Bienestar psicológico en estudiantes de danza contemporánea (Tesis de licenciatura). Pontificia Universidad Católica del Perú, Lima-Perú.

Ryff, C. (1989). Happiness is Everything, or Is It? Explorations on the Meaning of Psychological Well-Being. Journal of Personality and Social Psychology, 57, 1089-1081.

Ryff, C., y Keyes, C. (1995). The Structure of Psychological Well-Being Revisi- ted. Journal of Personality and Social Psychology, 69(4), 719-727.

Robles, J., Sánchez, A., y Galicia, I. (2011). Relación de bienestar psicológico, depresión y rendimiento académico en estudiantes de secundaria. Psicología Iberoamericana, 19(2), 27-37.

Sandin, B., y Chorot, P. (2003). Cuestionario de Afrontamiento del Estrés (CAE): Desarrollo y validación preliminar. $R e-$ vista de Psicopatología y Psicología Clínica 2003, 8(1), 39-54.

Serrano, M., y Flores, M. (2005). Estrés, respuestas de afrontamiento e ideación suicida en adolescentes. Psicología y Salud, 15(2), 221-230. Recuperado de http://inpsiquiatria.inteliglobe-mex. $\mathrm{com} / \mathrm{portal} / \mathrm{saludxmi} /$ biblioteca/estres/007_Estres_Respuestas_Afrontamiento.PDF

Velásquez, C., Mongotmery, W., Montero, V., Pomalaya, R., Dioses, A., Velásquez, N., Araki, R., y Reynoso, D. (2008). Bienestar psicológico, asertividad y rendimiento académico en estudiantes universitarios sanmarquinos. Revista de Investigación en Psicología, 11(2), 139-152. 